\title{
Elevated HTRA1 and HTRA4 in severe preeclampsia and their roles in trophoblast functions
}

\author{
CHUNHONG LIU $^{1 *}$, FENG XING $^{1 *}$, YUANYING HE ${ }^{1}$, SHANSHAN ZONG $^{2}$,

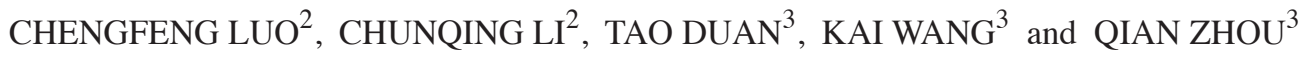 \\ ${ }^{1}$ Department of Obstetrics and Gynecology, Shanghai Tenth People's Hospital, Tongji University, \\ Shanghai 200072; ${ }^{2}$ Department of Obstetrics and Gynecology; ${ }^{3}$ Clinical and Translational Research Center, \\ Shanghai First Maternity and Infant Hospital, Tongji University, Shanghai 200040, P.R. China
}

Received December 11, 2017; Accepted June 28, 2018

DOI: $10.3892 / \mathrm{mmr} .2018 .9289$

\begin{abstract}
Aberrant gene expression during placental development may affect fetal growth and contribute to preeclampsia. The high-temperature requirement A (HTRA) family of proteins are serine proteases that may serve in the quality control of misfolded or mislocalized proteins. Recently, the potential involvement of HTRA1 and HTRA4 in the normal development of the placenta and in the pathogenesis of preeclampsia has been reported. The present study collected placental tissues from patients with severe preeclampsia and gestational age-matched control samples. The expression of HTRA1 and HTRA4 was analyzed using reverse transcription-quantitative polymerase chain reaction, western blotting and immunohistochemistry. The human trophoblast line HTR-8 was transfected with HTRA1 or HTRA4, and cell function was assessed. The present study also detected the expression of HTRA1 and HTRA4 in HTR-8/SVneo transfected cells under hypoxia $\left(1 \% \mathrm{O}_{2}\right)$ and further studied the effects of hypoxia on HTR-8 cell migration. HTRA1 and HTRA4 were mainly localized to the cytoplasm of syncytiotrophoblasts. The expression levels of the two genes were elevated in the placental tissues of patients with severe preeclampsia. Finally, it was determined in vitro that ectopic expression of HTRA1 and HTRA4 significantly attenuated HTR-8 cell migration, and elevated HTRA1 limited HTR-8 cell growth. Under hypoxic conditions, the expression levels of HTRA1 and HTRA4 improved significantly. It
\end{abstract}

Correspondence to: Dr Qian Zhou or Dr Kai Wang, Clinical and Translational Research Center, Shanghai First Maternity and Infant Hospital, Tongji University, 550 Shanghai South Road, Pudong New Area, Shanghai 200040, P.R. China

E-mail: shzhouqian@126.com

E-mail: kaiwangen@yahoo.com

${ }^{*}$ Contributed equally

Key words: preeclampsia, high-temperature requirement A1, high-temperature requirement A4, placenta, HTR-8, abnormally invasive placenta was hypothesized that the aberrant expression of HTRA1 or HTRA4 may be involved in the onset of preeclampsia, and increased HTRA1 or HTRA4 expression may affect trophoblast functions.

\section{Introduction}

Preeclampsia, generally defined as the development of hypertension and proteinuria after 20 weeks of gestation in a previously normotensive woman, remains a leading cause of fetal and maternal mortality and morbidity $(1,2)$. Preeclampsia is the second greatest cause of maternal mortality and affects $7-10 \%$ of pregnant women worldwide $(3,4)$. The etiology of preeclampsia begins in early pregnancy, when inadequate or defective placentation results in deficient maternal spiral artery remodeling and insufficient placental perfusion. This causes fetal hypoxia, which results in the release of multiple cytokines and growth factors into the maternal circulation, in turn leading to systemic maternal endothelial dysfunction, vascular inflammation, and the clinical manifestations of preeclampsia. Even though considerable data have been accumulated on the pathophysiology of preeclampsia, the precise underlying molecular mechanisms remain elusive.

During normal placentation, trophoblasts from the developing embryo invade the uterine sub-endometria and decidual spiral arteries, converting them into larger competent blood vessels to enhance blood flow to the embryo. Initial steps in this process require the trophoblast to detach from the extracellular matrix and invade the maternal arteries in a process tightly controlled by specific proteases. Recent studies have shown that shallow trophoblast invasion into the endometrium, resulting in insufficient spiral artery remodeling in the decidua, is the initial cellular pathology in preeclampsia, suggesting a possible molecular defect in proteolysis.

One class of proteases likely to be involved in placentation and preeclampsia is the high-temperature requirement $\mathrm{A}$ (HTRA) serine proteases, identified as important ATP-independent chaperones and cellular quality-control factors. Microarray data for the four known HTRA genes, after comparing normotensive pregnancy vs. preeclampsia, showed that HTRA1 and HTRA4 were significantly upregulated in preeclampsia compared with normal pregnancy (5). In a 
previous study of normal human placenta, HTRA1 expression was detected in trophoblast subtypes and in both uterine endometrial glands and decidual cells at the maternal-trophoblast interface during placentation (6). In mice, trophoblast invasion is coordinated with decidua regression and, as the process of placentation occurs, HtrA1 is highly expressed in both trophoblasts and in the decidua capsularis at its interface with the trophoblast during active cell invasion and regression (7). HtrA1 expression in human placenta and its role in relation to trophoblasts were also analyzed (8). Recently, increased expression of HTRA4 was reported in the sera and placentas of patients with preeclampsia, demonstrating that aberrant placental HTRA4 expression may be linked to the onset of preeclampsia (5). These observations indicate that increased HTRA1 or HTRA4 expression may be a manifestation of a functional defect in trophoblast migration and invasion and may be associated with preeclampsia.

Drawing on this background, we conducted an in-depth investigation of the expression of HTRA1 and HTRA4 in human placentas during physiologically normal and pathological pregnancies. Furthermore, we explored the role of HTRA1 and HTRA4 in cell cycle progression and migration in a trophoblast line.

\section{Materials and methods}

Ethical statement. The study protocol was approved by the Ethics Committees of the Shanghai First Maternity and Infant Hospital, Tongji University School of Medicine (Shanghai, China), and all participants provided written informed consent.

Patients and sample collection. A total of 20 placental biopsies, at different gestational ages in the third trimester, were obtained from patients with preeclamptic $(n=10)$ and normotensive pregnancies $(n=10)$. Severe preeclampsia was defined as patient blood pressure higher than $160 / 110 \mathrm{mmHg}$, platelets $<100,000 \mu 1$, elevated serum transaminase levels [alanine aminotransferase (ALT) or aspartate aminotransferase (AST)], persistent headache or other cerebral or visual disturbance, and persistent epigastric pain (9). The diagnosis of severe preeclampsia was based on strict criteria from the American College of Obstetricians and Gynecologists (ACOG 2013). Patient characteristics are shown in Table I.

All of the placental tissue samples in the study were obtained immediately after delivery. A central area of chorionic tissue was dissected, and the maternal decidua and amniotic membranes were removed. Sections of placental villi $(1 \times 1 \times 1 \mathrm{~cm})$ were taken from the four different central quadrants on the maternal face of the placenta. After prompt washing with sterile phosphate-buffered saline (PBS), tissues were immediately frozen and stored in liquid nitrogen until use for RNA and protein extraction. The remaining portions were fixed in $10 \%$ buffered formalin for immunohistochemistry (IHC).

$I H C$. Tissue biopsy specimens were embedded in paraffin and sectioned for IHC. After drying in an oven at $60^{\circ} \mathrm{C}$ for $2 \mathrm{~h}$, tissue sections were deparaffinized in xylene and dehydrated through a graded alcohol series. Endogenous peroxides were quenched by immersing the tissue sections in $3 \%$ hydrogen peroxide in methanol for $10 \mathrm{~min}$. After heat induction in citric acid for 15 min and blocking with $1 \%$ bovine serum albumin for $20 \mathrm{~min}$, the slides were incubated at $4{ }^{\circ} \mathrm{C}$ overnight with a rabbit polyclonal anti-HTRA1 at 1:100 dilution (ab65915; Abcam, Cambridge, UK) or anti-HTRA4 at 1:50 dilution (bs-20063R; Bioss, Beijing, China). After washing with PBS, the sections were subsequently incubated with IHC detection reagent (8114P; Cell Signaling Technology, Inc., Danvers, MA, USA) for $1 \mathrm{~h}$ at room temperature. Staining was completed by incubation with diaminobenzidine tetrahydrochloride, and tissues were counterstained with hematoxylin. Photomicrographs were taken with an inverted microscope.

Cell culture and transfection. The immortalized first-trimester human trophoblast line (HTR-8) (10), originally obtained from Dr. Charles H. Graham (Queen's University, Kingston, ON, Canada), has been reported to exhibit a number of characteristics similar to those of parental trophoblasts. The cells were cultured in Dulbecco's modified Eagle's medium: Nutrient Mixture F-12 (DMEM/F12) (SH30023; HyClone; GE Healthcare Life Sciences, Logan, UT, USA) supplemented with $10 \%(\mathrm{v} / \mathrm{v})$ fetal bovine serum (FBS) and antibiotics (100 U/ml penicillin and $100 \mathrm{mg} / \mathrm{ml}$ streptomycin) at $37^{\circ} \mathrm{C}$ in a $95 \%$ air and $5 \% \mathrm{CO}_{2}$ water-saturated atmosphere. To induce hypoxia, the cells were exposed to $1 \% \mathrm{O}_{2}, 5 \% \mathrm{CO}_{2}$ and $94 \% \mathrm{~N}_{2}$ in a multi-gas incubator (Sanyo, Osaka, Japan).

For transient expression of HTRA1 and HTRA4, HTR-8 cells were divided into three groups: Cells transduced with negative control adenovirus (HTR-8/vector), cells transduced with HTRA1-adenovirus (HTR-8/HTRA1) (AH891211; ViGene Biosciences, Rockville, MD, USA) or cells transduced with HTRA4-adenovirus (HTR-8/HTRA4) (AH894511; ViGene Biosciences). When HTR-8 cells were 50-70\% confluent, they were transduced with specific or negative control adenovirus for $6 \mathrm{~h}$, and these transfected cells were cultured in DMEM/F12 (HyClone; GE Healthcare Life Sciences) at $37^{\circ} \mathrm{C}$ for further analysis.

RNA isolation and reverse transcription-quantitative polymerase chain reaction ( $R T-q P C R)$. Total RNA was extracted from the placental villous tissues and cells using TRIzol and an RNA Simple Total RNA kit (DP419; Tiangen Biotech, Beijing, China) according to the manufacturer's instructions. RNA was quantified by UV absorption measurement and $1 \mu \mathrm{g}$ RNA was reverse transcribed into cDNA in a $20-\mu 1$ reaction volume using the PrimeScript RT reagent kit (FP205; Takara Bio, Inc., Otsu, Japan) for $15 \mathrm{~min}$ at $37^{\circ} \mathrm{C}, 5 \mathrm{sec}$ at $85^{\circ} \mathrm{C}$. RT-qPCR was performed using SYBR PreMix Ex Taq (Takara Bio, Inc.). The RT-qPCR reaction conditions were: Incubation at $95^{\circ} \mathrm{C}$ for $30 \mathrm{sec}$, followed by 40 cycles of $95^{\circ} \mathrm{C}$ for $15 \mathrm{sec}$ and $60^{\circ} \mathrm{C}$ for $20 \mathrm{sec}$. The PCR products were subjected to a melting curve analysis to confirm the amplification specificity. Levels of mRNA were normalized to $\beta$-actin and evaluated using the $2^{-\Delta \Delta \mathrm{Cq}}$ method (11). PCR primers for HTRA1, HTRA4, and the housekeeping gene $\beta$-actin were obtained from a previous report. The following primer sequences were used: HTRA1: 5'-GGACTACATCCAGACCGAC-3' and 5'-TGGGACTCC GTGAGGAAC-3'; HTRA4: 5'-GTCAGCACCAAACAG CG-3' and 5'-GGAGATTCCATCAGTCACCC-3'; and $\beta$-actin: 
Table I. Characteristics of subjects with normotensive pregnancies and severe preeclampsia.

\begin{tabular}{lccc}
\hline Variable & $\begin{array}{c}\text { Normotensive } \\
(\mathrm{n}=10)\end{array}$ & $\begin{array}{c}\text { sPE } \\
(\mathrm{n}=10)\end{array}$ & P-value \\
\hline Maternal age (years) & $29.4 \pm 1.1$ & $29.1 \pm 1.3$ & 0.86 \\
Gestational age (week) & $38.9 \pm 0.4$ & $38.0 \pm 0.6$ & 0.23 \\
Systolic BP (mmHg) & $109.7 \pm 3.3$ & $168.5 \pm 3.7$ & $<0.001$ \\
Diastolic BP (mmHg) & $69.5 \pm 2.2$ & $100.2 \pm 2.05$ & $<0.001$ \\
Fetal weight (g) & $3,435.6 \pm 81.3$ & $2,718.0 \pm 126.1$ & $<0.001$ \\
Proteinuria (g/24 h) & $0.12 \pm 0.02$ & $2.9 \pm 0.2$ & $<0.001$ \\
\hline
\end{tabular}

Data are presented as the mean \pm standard error of the mean. BP, blood pressure; sPE, serve preeclampsia.

\section{5'-AACTCCATCATGAAGTGTGACG-3' and 5'-GATCCA CATCTGCTGGAAGG-3'.}

Western blotting. Tissues and cells were lysed using radioimmunoprecipitation assay (RIPA) lysis buffer (P0013B; Beyotime Institute of Biotechnology, Haimen, China) containing protease inhibitors (10 $\mathrm{mM}$ EDTA and $4 \mathrm{mM}$ sodium pyrophosphate) and further lysed by sonication after pulverizing placental specimens in liquid nitrogen using a mortar and pestle. Following centrifugation, the protein concentration of each supernatant was determined with a bicinchoninic acid (BCA) Protein Assay kit (23225; Thermo Fisher Scientific, Inc., Waltham, MA, USA). Equal amounts of proteins $(100 \mu \mathrm{g})$ were boiled at $100^{\circ} \mathrm{C}$ for $10 \mathrm{~min}$, separated by sodium dodecyl sulfate polyacrylamide gel electrophoresis $(10 \%$ polyacrylamide) and electro-transferred onto polyvinylidene difluoride membranes. After blocking with 5\% non-fat milk in Tris-buffered saline [including $0.1 \%$ (w/v) Tween-20] at room temperature for $1 \mathrm{~h}$, membranes were first probed with rabbit polyclonal anti-HTRA1 (ab38610; Abcam) or with rabbit polyclonal anti-HTRA4 (ab65915; Abcam), followed by reprobing with an antibody against glyceraldehyde-3-phosphate dehydrogenase (GAPDH) (ab37168; Abcam) as a loading control. Proteins were detected using enhanced chemiluminescence (ECL) (NCI4106; Thermo Fisher Scientific, Inc.). Finally, the immunoreactive signals were quantified using a densitometer.

Proliferation assay. To assess proliferation rates, we used the BrdU incorporation assay to analyze DNA synthesis. After transfection for $30 \mathrm{~h}$, the cells were trypsinized, resuspended, and grown on 6 -well plates covered with $10 \times 10-\mathrm{mm}$ polylysine-coated coverslips. After incubating with $60 \mu \mathrm{M}$ BrdU reagent (KGA326; Sigma-Aldrich; Merck KGaA, Darmstadt, Germany) for $12 \mathrm{~h}$, the cells were fixed with cold acetone-methanol $(1: 1, \mathrm{v} / \mathrm{v})$ for $10 \mathrm{~min}$ and denatured with $4 \mathrm{~mol} / 1 \mathrm{HCl}$ for $10 \mathrm{~min}$, followed by three washes with PBS. The cells were incubated with $1 \mathrm{M}$ Tris- $\mathrm{HCl}(\mathrm{pH} 8.0)$ with mouse anti-BrdU (1:1,000; Sigma-Aldrich; Merck KGaA) and Cy3-labeled secondary antibody (1:300; Jackson ImmumoResearch Laboratories, Inc., West Grove, PA, USA). The BrdU+ cells were visualized by immunofluorescence. Nuclei were stained with $1 \mu \mathrm{g} / \mathrm{ml}$ DAPI (Dojindo Molecular Technologies, Inc., Kumamoto, Japan) and the images for cell counting were obtained from at least 10 randomly selected fields.
Cell cycle assay. A Cell Cycle Detection kit (KAG511; Keygen, Nanjing, China) was used to monitor the cell cycle. HTR-8 cells $\left(1 \times 10^{6}\right)$ were fixed in ice-cold $70 \%$ ethanol overnight at $4^{\circ} \mathrm{C} 48 \mathrm{~h}$ after transfection, and then incubated with $100 \mu \mathrm{g} / \mathrm{ml}$ RNase A at $37^{\circ} \mathrm{C}$ for $30 \mathrm{~min}$. After staining with $25 \mu \mathrm{g} / \mathrm{ml}$ propidium iodide for $30 \mathrm{~min}$, the cells were subjected to fluorescence-activated cell sorting and analyzed using Cell Quest Pro Software (BD Biosciences, Franklin Lakes, NJ, USA.

Migration assay. A transwell chamber assay was performed to analyze cell migration ability. HTR-8 cells $\left(5 \times 10^{4}\right)$ transfected with different adenoviruses for $30 \mathrm{~h}$ were trypsinized, resuspended, and seeded into the upper $8-\mu \mathrm{m}$ pore-size transwell chamber (BD Biosciences) with DMEM/F12 medium (300 $\mu \mathrm{l})$ containing $1 \% \mathrm{FBS}$, and the bottom chambers were filled with $800 \mu \mathrm{l}$ medium containing $10 \% \mathrm{FBS}$. After $16 \mathrm{~h}$ at $37^{\circ} \mathrm{C}$, cells that migrated to the lower chambers were stained with calcein AM (0.2 $\mu \mathrm{M}$; C3100MP; Invitrogen; Thermo Fisher Scientific, Inc.) for $30 \mathrm{~min}$ and visualized with an inverted microscope mounted with a charge-coupled device (CCD) camera. The cells were randomly counted under the microscope (magnification, x100) in four representative quadrants on each plate.

Statistical analysis. All experiments were carried out in three independent replicates. Data are expressed as the mean \pm standard error of the mean and differences between groups were determined using Student t-tests. $\mathrm{P}<0.05$ was considered to indicate a statistically significant difference. SPSS version 17.0 software (SPSS, Inc., Chicago, IL, USA) was used to analyze the data, and GraphPad Prism version 5.0 software (GraphPad Software, Inc., La Jolla, CA, USA) was used to create the graphs.

\section{Results}

Localization of HTRA1 and HTRA4 proteins in human placenta. First, we localized HTRA1 and HTRA4 proteins in human placenta tissues from normal and severe preeclamptic patients by IHC. In the third trimester, both HTRA1 (Fig. 1A and B) and HTRA4 (Fig. 1C and D) mainly localized to the cytoplasm of syncytiotrophoblasts in the placental villus. In addition, we observed weak staining for HTRA1 in the fetal vessel endothelium. Upregulation of the HtrA1 and HtrA4 in syncytiotrophoblasts from severe preeclampsia were confirmed with the use of quantitative analysis (Fig. 1E). 


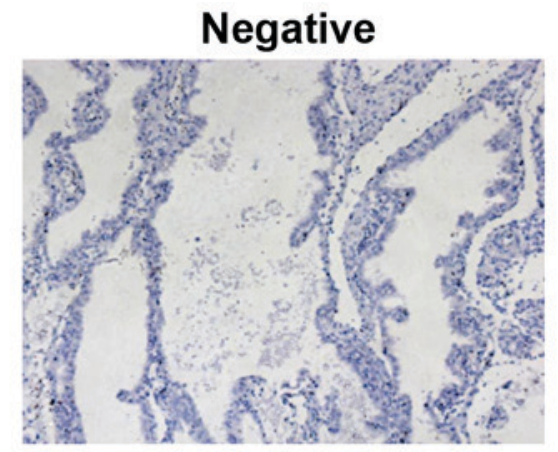

A

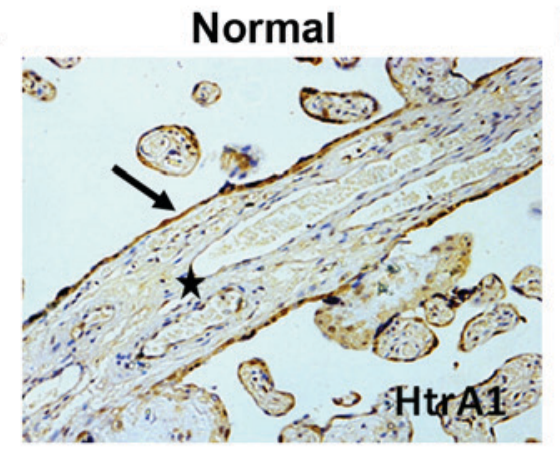

C

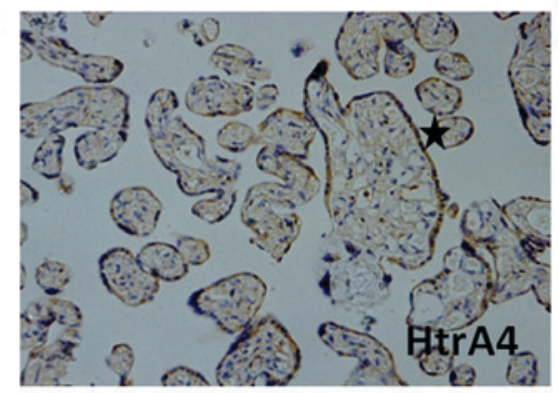

B
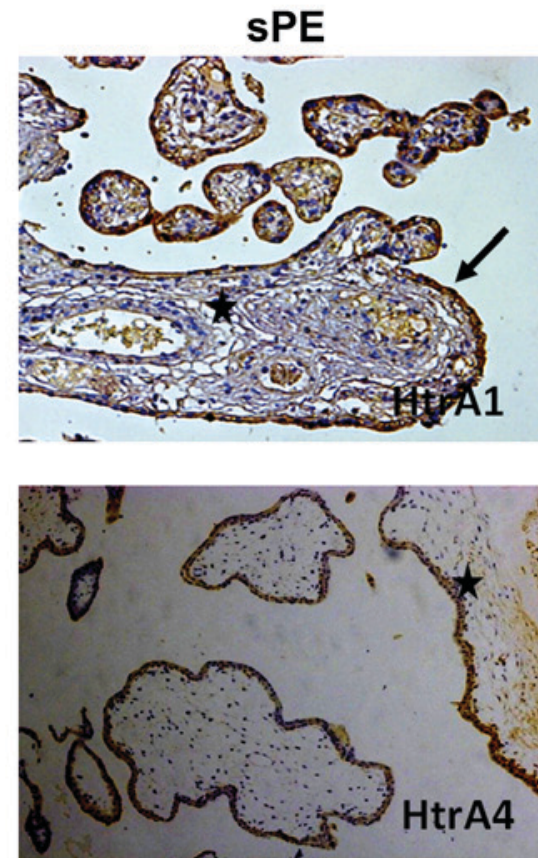

E
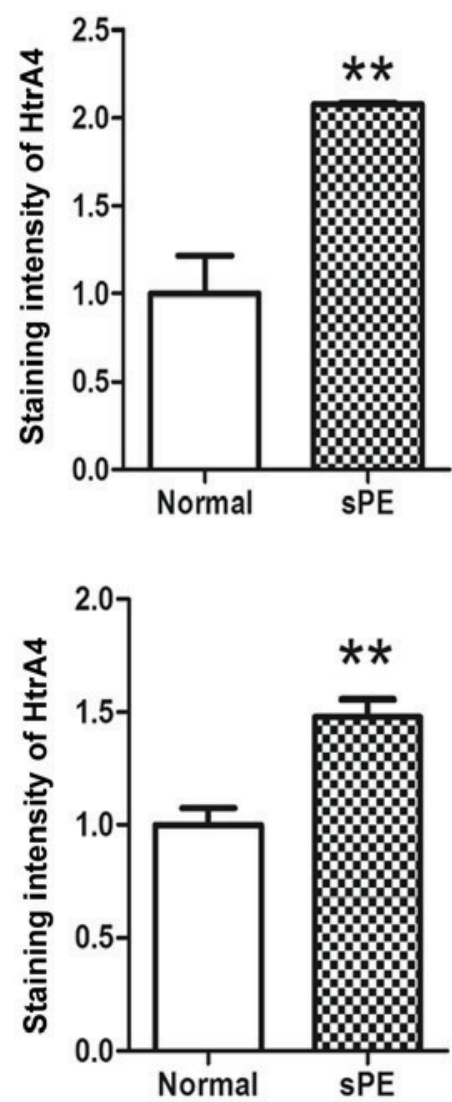

Figure 1. Representative HtrA1 and HtrA4 immunostaining of placental tissues from the third trimester of gestation (magnification, x200). Expression of HtrA1 and HtrA4 in villous trophoblasts from a normotensive placenta and a gestational age-matched severe preeclamptic placenta. (A and B) HtrA1 is mainly localized in the villous syncytiotrophoblast, the stroma of villi (indicated by arrows) and in the fetal vessel walls (indicated by stars). (C and D) HtrA4 localized to the villous syncytiotrophoblast (indicated by stars). (E) Stronger signal intensity of HtrA1 and HtrA4 were observed in the severe preeclampsia groups when compared with the normotensive groups. Data are shown as mean \pm standard error of the mean. ${ }^{* *} \mathrm{P}<0.01$ vs. normal. HtrA, high-temperature requirement A; sPE, severe preeclampsia group.

Upregulated expression of HTRAl and HTRA4 in human severe preeclamptic placentas. Next, RT-qPCR and western blotting were performed to compare the expression levels of HTRA1 and HTRA4 in different placental samples. We validated that the expression of HTRA1 and HTRA4 at both the mRNA (Fig. 2A) and protein (Fig. 2B and C) levels, after normalization with GAPDH, were significantly elevated in the severe preeclamptic placentas in the third trimester of gestation compared to the control group. In western blotting, both HTRA1 and HTRA4 antibodies detected a single band at $\sim 51 \mathrm{kDa}$ in HTR- 8 cells and in human placental tissues.

HTRA1 regulates proliferation and cell cycle in HTR-8 cells. Western blotting of HTR-8 cells derived from first-trimester trophoblast culture revealed that both HTRA1 and HTRA4 were minimally expressed. After transient transfection with control and specific adenoviruses, respectively, for 24, 48, 72, and $96 \mathrm{~h}$, western blotting indicated higher expression levels of HTRA1 and HTRA4 (Fig. 3A) in transfected cells.
To directly determine the effects of HTRA1 or HTRA4-induced HTR-8 cell proliferation, the rates of DNA synthesis in HTR-8/vector, HTR-8/HTRA1, and HTR-8/HTRA4 cells were quantified using BrdU incorporation assays. HTR-8/HTRA1 proliferation was reduced by $18 \%$ compared with control cells. However, ectopic HTRA4 expression had no effect on the growth of HTR-8 cells (Fig. 3B). Furthermore, flow cytometric analysis indicated that the percentage of HTR-8/HTRA1 cells in the G0/G1 phase increased significantly compared with that of control cells, concomitant with decreases in S-phase and G2/M-phase cells (Fig. 3C).

Both HTRA1 and HTRA4 attenuate migration of HTR-8 cells. To investigate whether HTRA1 and HTRA4 affect the migration activity of HTR-8 cells, transwell cell migration experiments were conducted. The mean counts of migrating cells in both the HTRA1 and HTRA4-transfected HTR-8 cells were significantly lower than in the control group (Fig. 3D). These observations indicate that increased HTRA1 and 
A

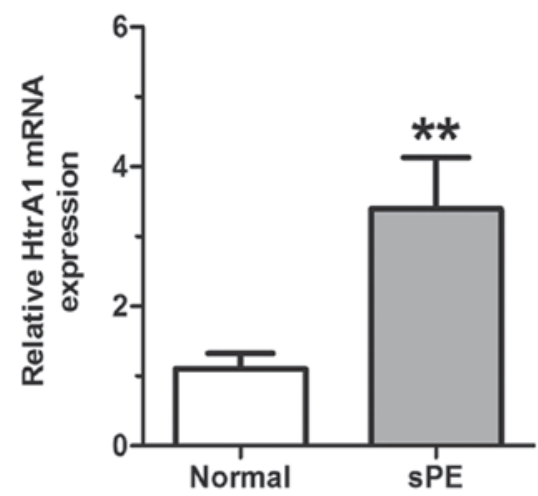

B
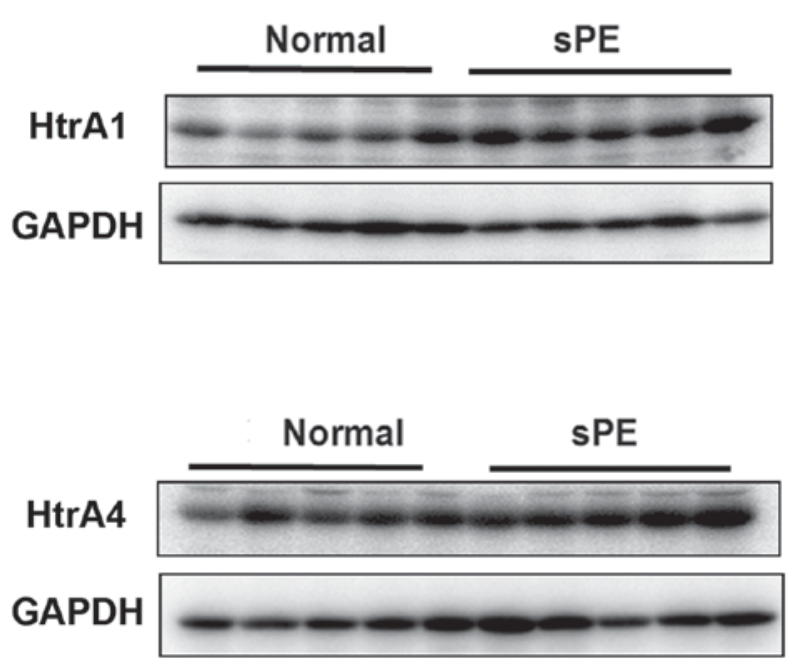

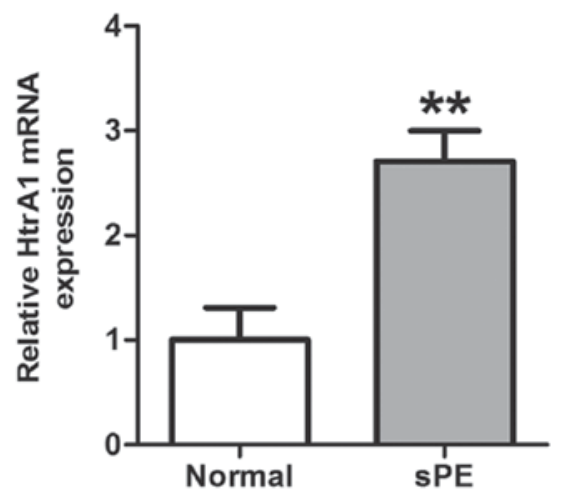

C
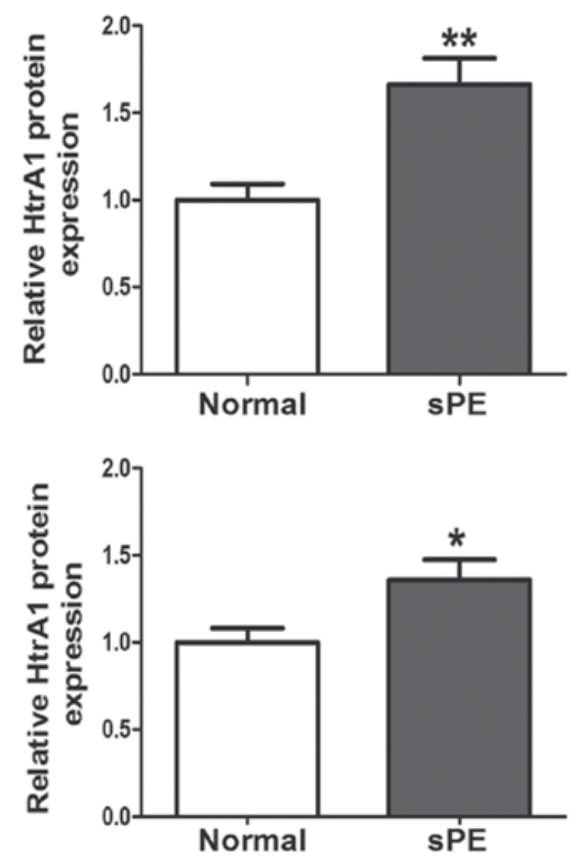

Figure 2. Differential expression of HtrA1 and HtrA4 in placental tissue samples from normotensive and severe preeclamptic pregnancies. (A) Reverse transcription-quantitative polymerase chain reaction analysis of HtrA1 and HtrA4. Data obtained from normotensive pregnancy and severe preeclampsia were compared. (B) The protein levels of HtrA1 or HtrA4 were normalized to GAPDH protein expression. Lanes 1-5, lysates from normotensive pregnancies; lanes 6-10, lysates from severe preeclampsia patients. (C) Quantitative western blotting results of HtrA1 and HtrA4 proteins between the normotensive and severe preeclampsia groups. Data are shown as the mean \pm standard error of the mean. ${ }^{*} \mathrm{P}<0.05$ and ${ }^{* *} \mathrm{P}<0.01$ vs. normal. HtrA, high-temperature requirement $\mathrm{A}$; sPE, severe preeclampsia group.

HTRA4 expression may inhibit trophoblast migration during placental development.

Hypoxia-induced HTRAl and HTRA4 expression and attenuated HTR-8 cell migration. As development of preeclampsia is tightly associated with placental hypoxia, HTR-8 cells were incubated for $0,16,24$, and $48 \mathrm{~h}$ under normoxic or hypoxic conditions, and the endogenous expression of HTRA1 and HTRA4 was determined by RT-qPCR and western blotting. Interestingly, our studies indicated that hypoxic treatment had a significantly positive effect on HTRA1 and HTRA4 levels in HTR-8 cells at 16 and $24 \mathrm{~h}$ (Fig. 4A).

Accordingly, we further investigated cell migration ability in hypoxia and discovered that the migration ability of HTR-8 cells was significantly impaired after hypoxic treatment for $26 \mathrm{~h}$. These results suggest that the inhibition of trophoblast migration by hypoxia may be due to the upregulation of HTRA1 and HTRA4 expression during placentation (Fig. 4B).

\section{Discussion}

Successful pregnancy depends on a coordinated series of gene expression in the placenta and embryo. Placentation requires trophoblasts to proliferate, migrate and invade the uterus and its spiral arterioles. The root cause of preeclampsia is reduced placental perfusion commonly caused by abnormal placentation and aberrant trophoblast functions.

In the present study, we have demonstrated that mRNA and protein levels of both HTRA1 and HTRA4 were upregulated in the villous tissues of placentas complicated preeclampsia by in late gestation, compared with the placentas of normotensive pregnant women. Consistent with the fact that HTRA1 and HTRA4 can be secreted, levels are also elevated in the sera of patients with preeclampsia $(5,12)$. These results independently confirmed our findings. In addition, HTRA4 was found to be upregulated in unexplained fetal growth restriction, which shares a common etiological pathway with preeclampsia; thus, 

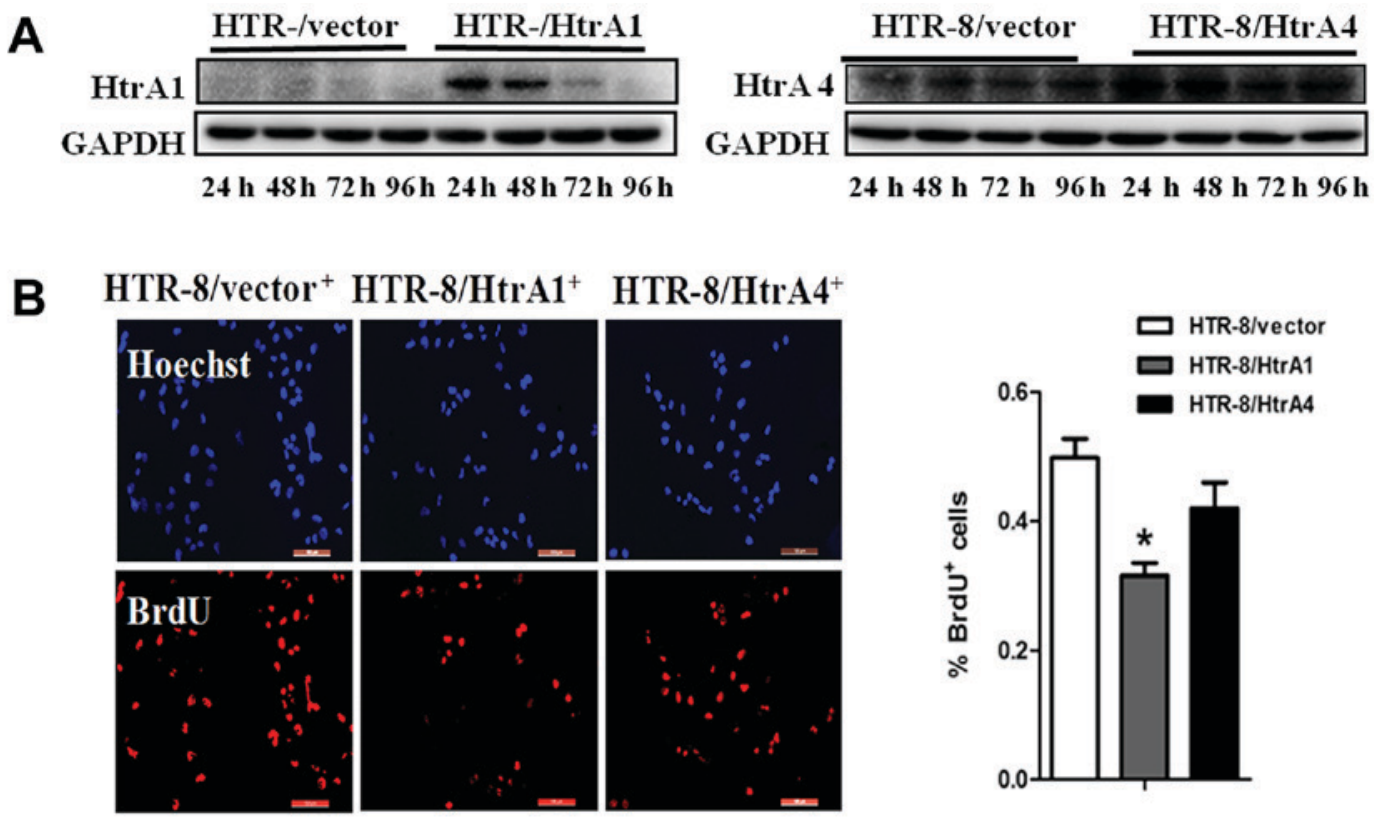

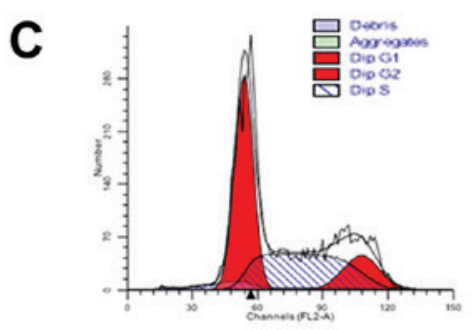

HTR-8/vector

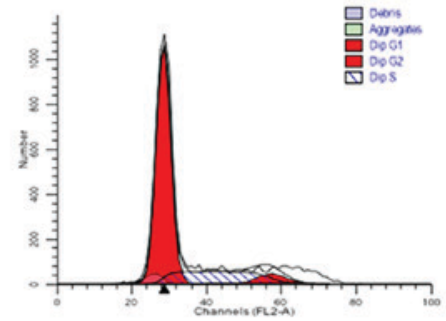

HTR-8/HtrA1

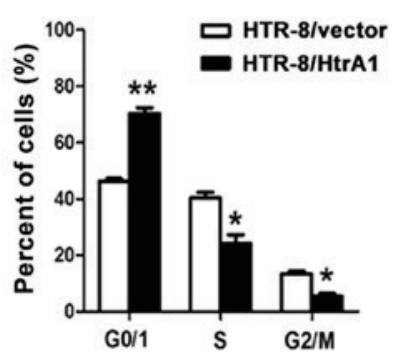

D
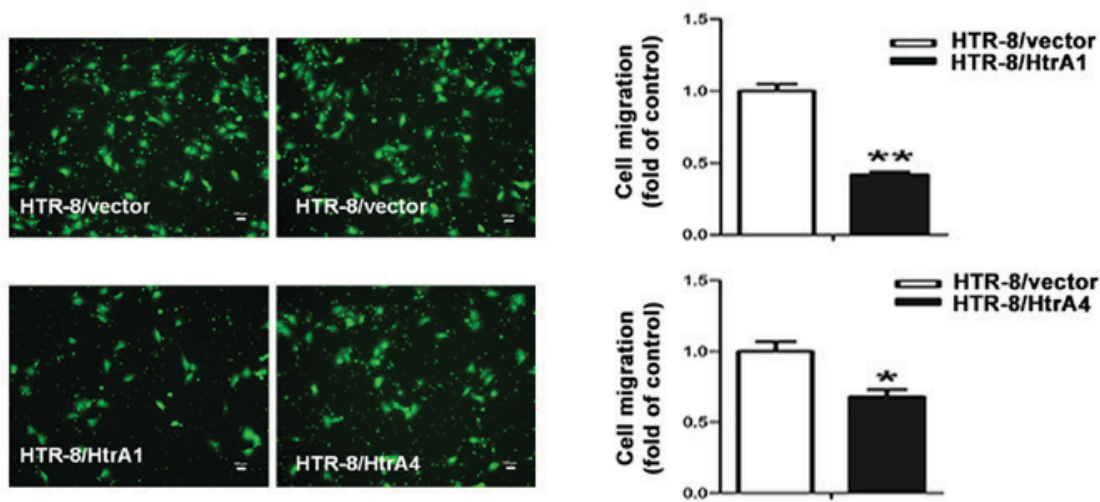

Figure 3. Western blotting for HtrA1 and HtrA4 in cells. (A) Expression of HtrA1 and HtrA4 in transfected HTR-8 cells was increased at 24,48 and 72 h when compared with the control groups. (B) Proliferating cells of HTR-8/vector, HTR-8/HtrA1, and HTR-8/HtrA4 were labeled with BrdU, and identified with anti-BrdU (red) and counterstained with Hoechst to visualize nuclei (blue). Scale bars, $100 \mu$ m. The percentage of BrdU-positive of HTR-8/HtrA1 in total Hoechst-stained cells was decreased when compared with the control groups. (C) Flow cytometric analysis demonstrated that the proportion of G0/1-phase cells increased, and the proportion of S and G2/M-phase cells decreased in the HTR-8/HtrA1 cells. Data represent the average percentage of cells in each phase of the cell cycle in three independent triplicate experiments. (D) The migratory activities of transfected cells were estimated based on the number of cells that migrated through the filter of the chamber (scale bars, $100 \mu \mathrm{m}$; magnification, x100). Quantitation indicated that the number of migrating cells in the HtrA1 and HtrA4-transfected groups was less than the number observed in the control groups. Results are presented as the mean \pm standard error mean $\left(\mathrm{n}=3\right.$ ). ${ }^{*} \mathrm{P}<0.05$ and ${ }^{* *} \mathrm{P}<0.01$ vs. HTR-8/vector. HtrA, high-temperature requirement A; BrdU, 5-bromo-2'-deoxyuridine.

HTRA4 may trigger preeclampsia via a pathway common to fetal growth restriction.

Our results showed that ectopic expression of HTRA1 or HTRA4 in HTR-8 cells inhibited their migration. Interestingly, several studies have indicated that HtrA1, as a tumor suppressor downregulated in ovarian cancer (13) and in melanoma metastases, induces cell death and inhibits proliferation and invasion (14). Thus, HTRA1 seems to have two paradoxical roles. HTRA family proteases share similar molecular architecture. Human HTRA1 and HTRA4 are composed of variable $\mathrm{N}$ termini, a protease domain, and one PDZ domain. Their role in cell migration is probably not dependent on their protease activity, but on the PDZ domain or the $\mathrm{N}$ terminal regions, which contain signal peptides and 
A

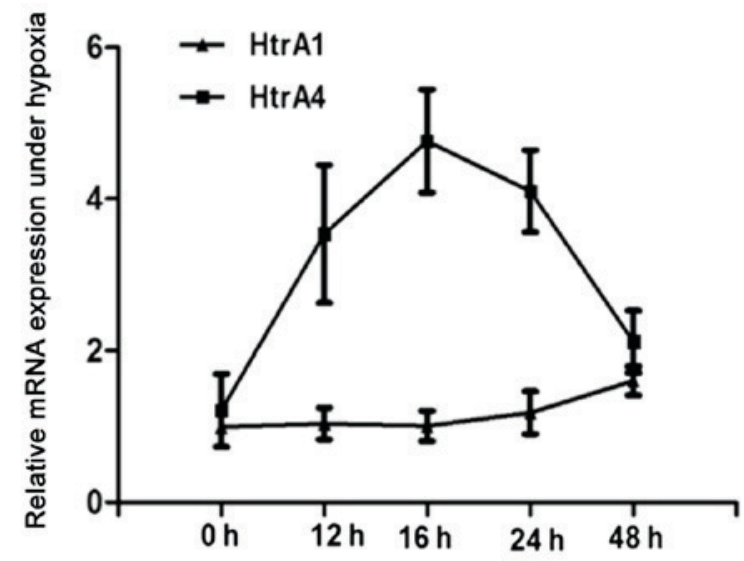

B
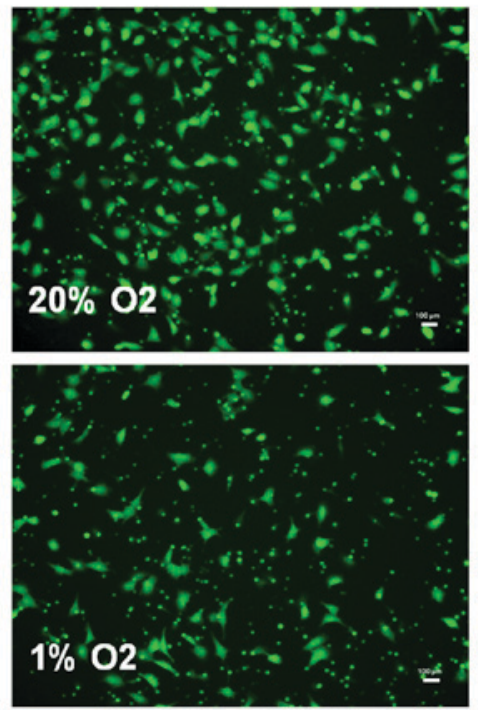

Oh $\quad 16 \mathrm{~h} \quad 24 \mathrm{~h} \quad 48 \mathrm{~h}$

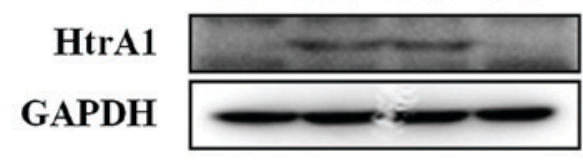

Oh $\quad 16 \mathrm{~h} \quad 24 \mathrm{~h} 48 \mathrm{~h}$
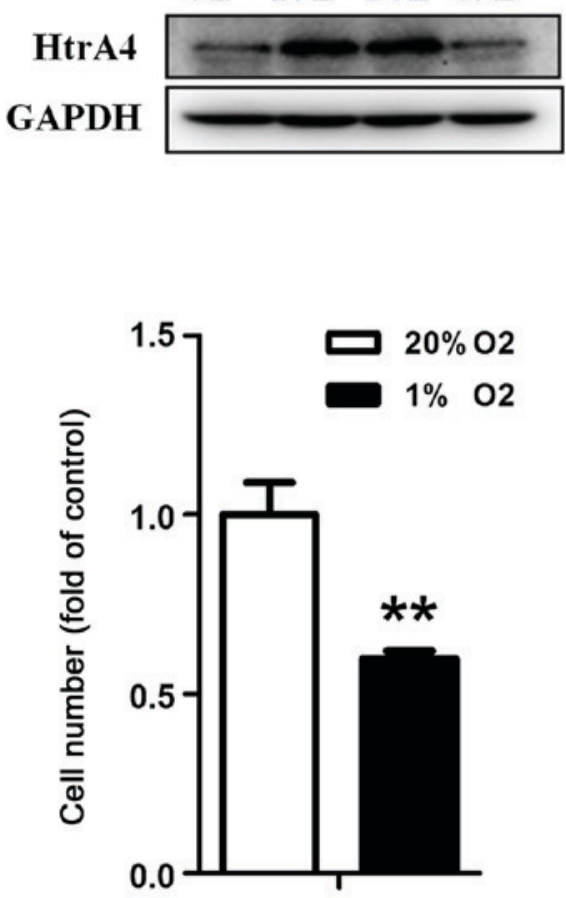

Figure 4. Effects of hypoxia on HtrA1 and HtrA4 expression, and on cell migration in HTR-8 cells. (A) Expression of HtrA1 and HtrA4 increased in HTR-8 cells under hypoxia $\left(1 \% \mathrm{O}_{2}\right)$ when compared with normoxia $\left(20 \% \mathrm{O}_{2}\right)$, which peaked at $16 \mathrm{~h}$. (B) The number of migrating cells in the hypoxia group was less than those observed in the control group. Scale bars, $100 \mu \mathrm{m}$. Results are presented as the mean \pm standard error of the mean $(\mathrm{n}=3){ }^{* * *} \mathrm{P}<0.001 \mathrm{vs}$. normoxia $\left(20 \% \mathrm{O}_{2}\right)$. HtrA, high-temperature requirement A.

a fragment of insulin-like growth factor binding protein (15). Moreover, a wide variety of hormones differ in variety and quantity during pregnancy. Therefore, to investigate the roles of these domains in trophoblast migration in the placenta, further experiments involving various deletion constructs of HTRA1 and HTRA4 are needed.

We identified HTRA1 as an inhibitory factor in trophoblast proliferation and progression past the G1/S checkpoint of the cell cycle. Previous studies have implicated intracellular HTRA1 in the proliferation of multiple cell types, and it may mediate these effects by inhibiting a number of proteins such as tuberous sclerosis complex 2 (TSC-2), participating in increasing cell growth and proliferation (16), insulin-like growth factor binding protein 5 (17), and transforming growth factor- $\beta$. Therefore, HTRA1 may suppress trophoblast proliferation through its effects on cell cycle progression.

Furthermore, we demonstrated that hypoxia $\left(\begin{array}{ll}1 \% & \mathrm{O}_{2}\end{array}\right)$ induced significant overexpression of both HTRA1 and HTRA4 and inhibited the chemotactic cell migration of HTR- 8 cells. The upregulation of HTRA1 and HTRA4 expression may be secondary to placental ischemia and hypoxia in preeclamptic pregnancies. However, due to complicated alterations of various factors in trophoblasts in hypoxia, it is uncertain whether overproduction of HTRA1 or HTRA4 directly leads to defects in HTR-8 cell migration. Oxidative stress and endoplasmic reticulum stress in trophoblasts play key intermediary roles in generating preeclampsia (18-20). Hypoxia may precipitate endoplasmic reticulum stress and lead to increased generation of reactive oxygen species (21). HTRA1 does not appear to be inducible by heat shock, but may be regulated by a more general stress pathway (22). HTRA1 or HTRA4, as essential chaperones, may be upregulated to stabilize misfolded or mislocalized proteins to enhance cell survival in preeclamptic placentas. Therefore, more experiments on HTRAl and HTRA4 are needed to determine their roles in trophoblast migration during hypoxia.

In conclusion, HTRA1 and HTRA4 are upregulated in severe preeclamptic placentas, which may be linked to disease onset. However, the specific mechanisms responsible for the elevation of HTRA1 or HTRA4 in preeclampsia and their roles in trophoblasts under hypoxia are not yet understood. Whether overexpression may be a primary step or secondary to placental ischemia and hypoxia or abnormal cytokine expression remains unclear. Thus, further studies are needed 
to elucidate the roles of HTRA1 and HTRA4 proteins in trophoblast function and to investigate the potential therapeutic utility of targeting this pathway.

\section{Acknowledgements}

The authors would like to thank Mrs. Xin Luo, Mrs. Huihui Wang,Mrs. Fangyuan Wang,Mrs. Yifei Chen and Mrs. Hui Wang (Experimental Center of International Peace Maternity \& Child Health Hospital Affiliated to Shanghai Jiao Tong University School of Medicine) for their technical assistance.

\section{Funding}

The present study was supported in part by a grant from the National Natural Science Foundation of China (grant no. 81771659).

\section{Availability of data and materials}

All data generated or analyzed during this study are included in this published article.

\section{Authors' contributions}

QZ and KW obtained funding and designed the present study. $\mathrm{ChL}$ and FX carried out the experiments and drafted the manuscript. YH, SZ, CfL, CqL and TD acquired, analyzed and interpreted the data. TD supervised the enrollment of patients, collected clinical data and revised the manuscript for important intellectual content.

\section{Ethics approval and consent to participate}

The present study was approved by the Ethics Committees of the Shanghai First Maternity and Infant Hospital, Tongji University School of Medicine (Shanghai, China), and all participants provided written informed consent.

\section{Patient consent for publication}

The patients provided written informed consent prior to participating in the present study.

\section{Competing interests}

All authors declare that they have no any conflict of interests.

\section{References}

1. Sibai BM, Caritis SN, Thom E, Klebanoff M, McNellis D Rocco L, Paul RH, Romero R, Witter F, Rosen M, et al: Prevention of preeclampsia with low-dose aspirin in healthy, nulliparous pregnant women. The National Institute of Child Health and Human Development Network of Maternal-Fetal Medicine Units. N Engl J Med 329: 1213-1218, 1993.

2. Levine RJ, Hauth JC, Curet LB, Sibai BM, Catalano PM, Morris CD, DerSimonian R, Esterlitz JR, Raymond EG, Bild DE, et al: Trial of calcium to prevent preeclampsia. N Engl J Med 337: 69-76, 1997.

3. Chappell LC, Enye S, Seed P, Briley AL, Poston L and Shennan AH: Adverse perinatal outcomes and risk factors for preeclampsia in women with chronic hypertension: A prospective study. Hypertension 51: 1002-1009, 2008.
4. National high blood pressure education program working group report on high blood pressure in pregnancy. Am J Obstet Gynecol 163: 1691-1712, 1990.

5. Inagaki A, Nishizawa $\mathrm{H}$, Ota S, Suzuki M, Inuzuka $H$, Miyamura H, Sekiya T, Kurahashi $H$ and Udagawa $Y$ : Upregulation of HtrA4 in the placentas of patients with severe pre-eclampsia. Placenta 33: 919-926, 2012.

6. Nie G, Hale K, Li Y, Manuelpillai U, Wallace EM and Salamonsen LA: Distinct expression and localization of serine protease HtrA1 in human endometrium and first-trimester placenta. Dev Dyn 235: 3448-3455, 2006.

7. Nie GY, Li Y and Salamonsen LA: Serine protease HtrA1 is developmentally regulated in trophoblast and uterine decidual cells during placental formation in the mouse. Dev Dyn 233: 1102-1109, 2005.

8. Ajayi F, Kongoasa N, Gaffey T, Asmann YW, Watson WJ, Baldi A, Lala P, Shridhar V, Brost B and Chien J: Elevated expression of serine protease HtrA1 in preeclampsia and its role in trophoblast cell migration and invasion. Am J Obstet Gynecol 199: 557.e1-e10, 2008.

9. American College of Obstetricians and Gynecologists; Task Force on Hypertension in Pregnancy: Hypertension in pregnancy. Report of the American College of Obstetricians and Gynecologists' Task Force on Hypertension in Pregnancy. Obstet Gynecol 122: 1122-1131,2013.

10. Graham CH, Hawley TS, Hawley RG, MacDougall JR, Kerbel RS, Khoo N and Lala PK: Establishment and characterization of first trimester human trophoblast cells with extended lifespan. Exp Cell Res 206: 204-211, 1993.

11. Livak KJ and Schmittgen TD: Analysis of relative gene expression data using real-time quantitative PCR and the 2(-Delta Delta $\mathrm{C}(\mathrm{T})$ ) method. Methods 25: 402-408, 2001.

12. Lorenzi T, Marzioni D, Giannubilo S, Quaranta A Crescimanno C, De Luca A, Baldi A, Todros T, Tranquilli AL and Castellucci M: Expression patterns of two serine protease HtrA1 forms in human placentas complicated by preeclampsia with and without intrauterine growth restriction. Placenta 30: 35-40, 2009.

13. Chien J, Staub J, Hu SI, Erickson-Johnson MR, Couch FJ, Smith DI, Crowl RM, Kaufmann SH and Shridhar V: A candidate tumor suppressor HtrA1 is downregulated in ovarian cancer. Oncogene 23: 1636-1644, 2004.

14. Baldi A, De Luca A, Morini M, Battista T, Felsani A, Baldi F, Catricalà C, Amantea A, Noonan DM, Albini A, et al: The HtrA1 serine protease is down-regulated during human melanoma progression and represses growth of metastatic melanoma cells. Oncogene 21: 6684-6688, 2002.

15. Oh Y, Nagalla SR, Yamanaka Y, Kim HS, Wilson E and Rosenfeld RG: Synthesis and characterization of insulin-like growth factor-binding protein (IGFBP)-7. Recombinant human mac25 protein specifically binds IGF-I and -II. J Biol Chem 271: 30322-30325, 1996.

16. Campioni M, Severino A, Manente L, Tuduce IL, Toldo S, Caraglia M, Crispi S, Ehrmann M, He X, Maguire J, et al: The serine protease HtrA1 specifically interacts and degrades the tuberous sclerosis complex 2 protein. Mol Cancer Res 8: 1248-1260, 2010.

17. Hou J, Clemmons DR and Smeekens S: Expression and characterization of a serine protease that preferentially cleaves insulin-like growth factor binding protein-5. J Cell Biochem 94: 470-484, 2005

18. Redman CW and Sargent IL: Latest advances in understanding preeclampsia. Science 308: 1592-1594, 2005.

19. Yung HW, Calabrese S, Hynx D, Hemmings BA, Cetin I, Charnock-Jones DS and Burton GJ: Evidence of placental translation inhibition and endoplasmic reticulum stress in the etiology of human intrauterine growth restriction. Am J Pathol 173: 451-462, 2008

20. Roberts JM and Hubel CA: Is oxidative stress the link in the two-stage model of pre-eclampsia? Lancet 354: 788-789, 1999.

21. Burton GJ, Yung HW, Cindrova-Davies T and Charnock-Jones DS: Placental endoplasmic reticulum stress and oxidative stress in the pathophysiology of unexplained intrauterine growth restriction and early onset preeclampsia. Placenta 30 (Suppl A): S43-S48, 2009.

22. Ly DH, Lockhart DJ, Lerner RA and Schultz PG: Mitotic misregulation and human aging. Science 287 : 2486-2492, 2000 . 